\title{
A small error in Thurstone's length-difficulty function
}

WILLIAM BOGARTZ

UNIVERSITY OF ILLINOIS AT CHICAGO CIRCLE

It is shown that Thurstone's well known square-root function relating length of a paired-associate list with trials to criterion was the result of a minor oversight. Length-difficulty relationships actually implied by Thurstone's learning theory are derived and discussed.

Thurstone's (1930) derivation of "the learning function" is perhaps of importance today only as one of the earliest mathematical theories of learning. Nevertheless, one result which purports to have beendeduced from this theory has achieved wide currency in the verbal learning literature (e.g., McGeoch \& Irion, 1960; Deese, 1967), namely, the proposition that the number. of repetitions of a paired-associate list necessary to reach a learning criterion is proportional, approximately, to the square root of the list length. It will be shown here that this result does not, in fact, follow from Thurstone's theory, but was incorrectly derived and uncritically accepted, insofar as its derivation is concerned. That the error remained unchallenged for so long may serve as an object lesson for psychologists who give repeated citation to conclusions in the literature without careful evaluation of their foundations.

The theory, in brief, is as follows. In any learning situation, there are, at a given time, a set of $s$ successful acts and another of e erroneous acts, whose respective probabilities, $p$ and $q$, are just the proportions of the total number of acts which are in each set at that time. The essential assumption is that the rates of change of $s$ and $e$ with respect to time ( $t)$ are proportional to their probabilities, with the constants of proportionality equal and opposite in sign. The development proceeds as follows:

(1) $\mathrm{p}=\mathrm{s} /(\mathrm{s}+\mathrm{e})$

(2) $q=e /(s+e)$

(3) $\mathrm{ps}+\mathrm{pe}=\mathrm{s}$

(4) $\mathrm{de} / \mathrm{dt}=-\mathrm{kq}$

(5) $\mathrm{de} / \mathrm{dt}=-\mathrm{ke} /(\mathrm{s}+\mathrm{e})$

(6) $\mathrm{ds} / \mathrm{dt}=\mathrm{kp}$

(7) $\mathrm{ds} / \mathrm{dt}=\mathrm{ks} /(\mathrm{s}+\mathrm{e})$

(8) $\mathrm{ds} / \mathrm{de}=-\mathrm{s} / \mathrm{e}$

(9) $\int \mathrm{ds} / \mathrm{s}+\int \mathrm{de} / \mathrm{e}=0$

Integrating,

(10) $\log s+\log e=\log m$

(11) $s e=m$ (a constant)

After expressing everything in terms of $p$ and integrating, Thurstone finally obtains

(12) $(2 p-1)\left(p-p^{2}\right)^{-1 / 2}=k^{-1 / 2}+\left(2 p_{0}-1\right)\left(2 p_{0}-p_{0}^{2}\right)^{-1 / 2}$ where $p_{0}$ is the value of $p$ at $t=0$. This is the learning function, in implicit form. It is curious that apparently no one has ever bothered to solve it explicitly for p. With no further apology, the solution is:

(13) $\mathrm{p}=1 / 2+\mathrm{x}\left(4 \mathrm{x}^{2}+16\right)^{-1 / 2}$

where $x=k t m-1 / 2+\left(2 p_{0}-1\right)\left(p_{0}-p_{0}^{2}\right)^{-1 / 2}$.

In applying the foregoing to paired-associate learning, Thurstone makes, substantially, the following assumptions (the introduction of an "attention span" constant will be omitted as an irrelevant complication). Since there are $\mathrm{n}^{2}$ possible pairs which can be formed from a set of $n$ stimuli and $n$ responses, and $n$ of these are correct in a paired-associate task, leaving $n(n-1)$ incorrect pairs, then at the beginning of the task, $s=n, e=n(n-1)$, and $m=n^{2}(n-1)$.

Equation (12) may be written

(14) $\mathrm{Z}=\mathrm{ktm}^{-1 / 2}+\mathrm{Z}_{0}$, so that

(15) $t=m^{1 / 2} k^{-1}\left(Z-Z_{0}\right)$.

If learning always proceeds to the same fixed value of $p$ (probability criterion), then $Z$, being a function of $p$ only, is also constant when the criterion value of $p$ has been reached. Substituting the value of $m$ obtained above, and also taking $\mathrm{Z}_{0}$ as constant, we have (16) $\mathrm{t}=\mathrm{ck}^{-1} \mathrm{n}(\mathrm{n}-1)^{1 / 2}$.

Since a repetition of the entire list takes $n$ time units, the number of repetitions (R) to a probability criterion is

(17) $\mathrm{R}=\mathrm{ck}^{-1}(\mathrm{n}-1)^{1 / 2}$

Some of the difficulties with Thurstone's analysis of this problem are strictly matters of assumption and, as such, cannot be gainsaid, but shouid be understood. Among these are the assumed constancy of the rate parameter, $k$, for different list lengths, and the choice of a probability criterion for comparing them. The variable $p$, under Thurstone's interpretation, refers to probability of a single correct response, not that of a perfect run through the entire list. Since the latter would be a function of $n$, the analysis is not applicable to trials to criterion in the usual type of pairedassociate learning experiment.

These are not the most telling points, however. Even if the model is correctly applied in a way consistent with Thurstone's analysis, there is an error in his derivation of the length-difficulty function. Because the quantity, $z_{0}=\left(2 p_{0}-1\right)\left(p_{0}-p_{0}^{2}\right)^{-1 / 2}$, arose as a constant of integration, it was natural to take it as constant 
in analyzing the paired-associate task. As long as one is dealing with a single learning function, this is correct. In the context of variable list length, however, it is entirely inappropriate, and leads to a function of appreciably different character from the one which should have been obtained.

The point is that the assumptions about initial values of $\mathbf{s}$ and $e$ which generated the value of $m$ used in the derivation also specify, by definition, the value of $p_{0}$. and therefore $Z_{0}$. That is,

(18) $p_{0}=s_{0} /\left(s_{0}+e_{0}\right)=n / n^{2}=1 / n$, whence,

(19) $Z_{0}=-(n-2)(n-1)^{-1 / 2}$

Substituting this value into Equation (12), we have, for the correct length-difficulty function,

(20) $t=n k^{-1}\left[c(n-1)^{1 / 2}+n-2\right]$, or

(21) $\mathrm{R}=\mathrm{k}^{-1}\left[\mathrm{c}(\mathrm{n}-1)^{1 / 2}+\mathrm{n}-2\right]$.

Actually, Thurstone was aware of the dependence of $\mathrm{Z}_{0}$ on $\mathrm{n}$, in principle, but regarded the function obtained by his method as an approximation. That this is not so will be clear from comparison of the first derivatives of Thurstone's function and the corrected version. The slope of the former is proportional to $(n-1)^{-1 / 2}$, which approaches zero with increasing $n$. This means that the curve comes closer and closer to a horizontal line, or that increasing length has in the limit no further effect upon the number of repetitions to a probability criterion. In contrast, the corrected function (21) has a first derivative proportional to $(c / 2)(n-1)^{-1 / 2}$ +1 . That is to say, although this function, like Thurstone's, is negatively accelerated, it is asymptotically directly proportional to $\mathrm{n}$. In the limit, increasing length uniformly increases the number of repetitions to criterion. It is easy to see that the two functions are quite different in their theoretical implications.

It is also interesting to consider the length-difficulty function which arises if we require that learning proceed to a constant expected number of errors, rather than a constant probability, this being a closer approximation to the usual type of verbal learning criterion (cf., Bogartz \& Headrick, 1965). That is, suppose that the process terminates, for a given list length, $n$, when $n(1-p)=c$, or $p=(n-c) / n$. Substituting this value in Equation (12), we obtain

(22) $R=k^{-1}\left[(n-2 c)(n-1)^{1 / 2}\left(c n-c^{2}\right)^{-1 / 2}+n-2\right]$.
This function quickly approaches a straight line as $n$ increases. For either a probability criterion or an error criterion, therefore, the theory predicts an asymptotically linear relationship between length of list and the number of repetitions.

Continuing in the spirit of Thurstone's analysis, and recognizing that in the case of a probability criterion for paired associate learning $n$ entered into the lengthdifficulty function only through its determination of $p_{0}$. while with an error criterion, $n$ appeared also in the terminal value of $p$, we may derive expressions for each of these cases for a task of length $n$, but with $r$ different response alternatives for every stimulus. This would include verbal discrimination tasks, for example. To accomplish this, assume that initially, $p_{0}=1 / r$, $s_{0}=n$, and that the final value of $p$ is either $c$ (constant), for the probability criterion (Equation 23 ), or $(n-c) / n$, as in the derivation of (22), for the error criterion (Equation 24). Substituting these values in Equation (12), we have

(23) $\mathrm{R}=\mathrm{k}^{-1} \mathrm{c}(\mathrm{r}-1)^{1 / 2}+\mathrm{r}-2$

(24) $R=k^{-1}(n-2 c)(r-1)^{1 / 2}\left(c n-c^{2}\right)^{-1 / 2}+r-2$.

Note that Equation (23) implies that for an $n$ item, $r$ alternative discrimination task, the number of repetitions to a probability criterion, i.e., until a given mean proportion of correct responses is to be expected, is independent of length. Furthermore, for fixed $n$ and variable $r$, both types of criterion produce the same form of function.

In conclusion, quite aside from the merits of Thurstone's learning theory as such, it is clear that all applications of his original length-difficulty function to experiments in the verbal learning literature have been quite unfounded, as far as that theory is concerned. These remarks were meant to elucidate the lengthdifficulty relationships actually implied by Thurstone's theory, and to correct any misconceptions of it which still remain.

\section{References}

BOGARTZ, W., \& HEADRICK, ARLENE. The length-difficulty law of serial learning: An empirical function and a theoretical derivation. Psychon. Sci, 1965, 3, 357-358.

DEESE, J., \& HULSE, S. H. The psychology of learning. New York: McGraw-Hill, 1967.

MOGEOCH, J. A., \& IRION, A. L. The psychology of human learning. New York: Longmans, Green \& Co., 1960.

THURSTONE, L. L. The learning function. J. gen. Psychol, 1930, 3, 469-493. 